\title{
The Mitochondrial Peptide Humanin Targets, but does not Denature Amyloid Oligomers in Type II Diabetes
}

\author{
Zachary A. Levine, ${ }^{1,2,{ }^{*}}$ Kazuki Teranishi, ${ }^{3,4}$ Alan K. Okada, ${ }^{3,4, \$}$ \\ Ralf Langen, ${ }^{3,4}$ and Joan-Emma Shea ${ }^{5,6}$
}

1. Department of Pathology

Yale School of Medicine, New Haven, CT, 06520, USA

2. Department of Molecular Biophysics \& Biochemistry, Yale University, New Haven, CT 06520, USA

3. Department of Biochemistry and Molecular Biology University of Southern California, Los Angeles, CA 90033, USA

4. Zilkha Neurogenetic Institute, Keck School of Medicine University of Southern California, Los Angeles, CA 90033, USA

5. Department of Chemistry and Biochemistry, University of California Santa Barbara, Santa Barbara, CA 93106, USA

6. Department of Physics, University of California Santa Barbara, Santa Barbara, CA 93106, USA

\section{§Present Address:}

Department of Emergency Medicine

Regions Hospital, St. Paul, MN 55101, USA

\section{* Corresponding author:}

Zachary A. Levine

Department of Pathology

Department of Molecular Biophysics \& Biochemistry

Yale University, New Haven, CT 06520, USA

Email: zachary.levine@yale.edu 


\section{SUPPORTING INFORMATION}
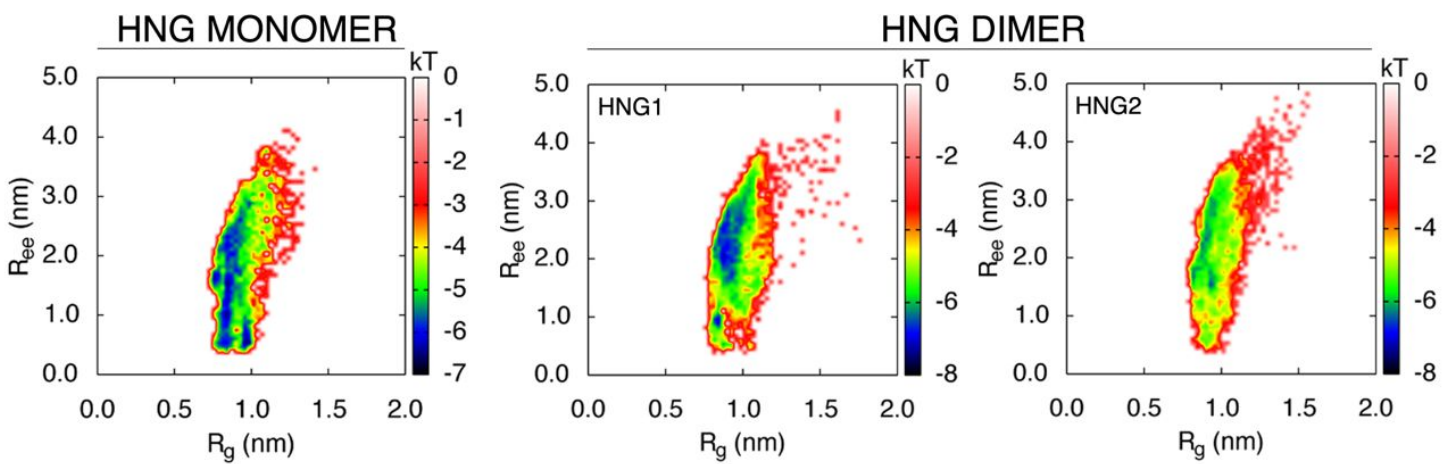

IAPP MONOMER
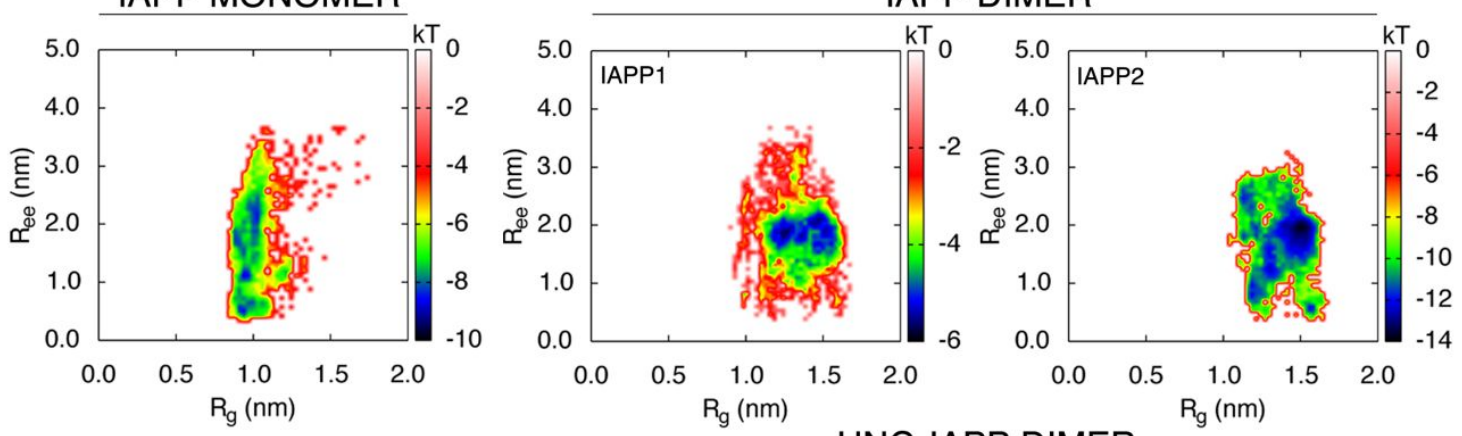

HNG-IAPP DIMER
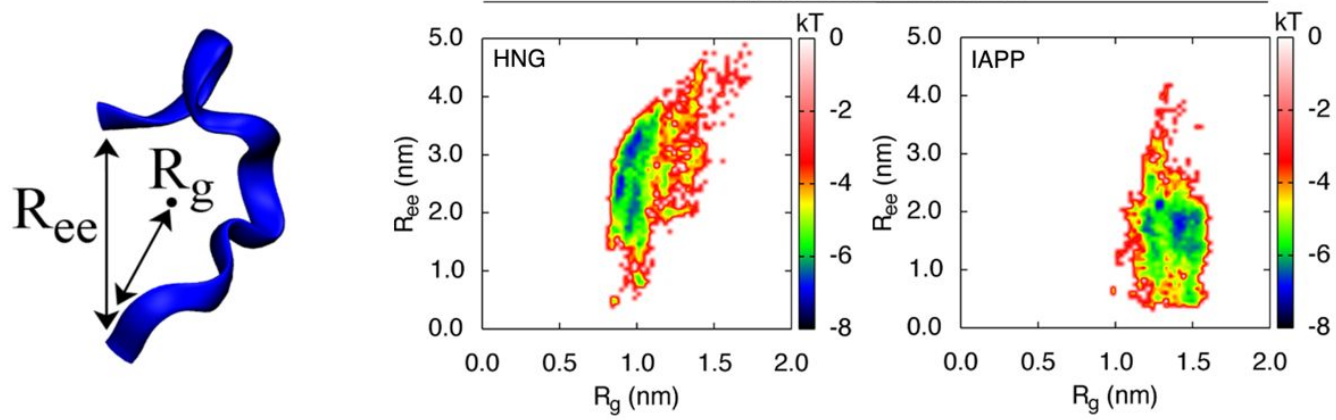

2IAPP-1HNG TRIMER
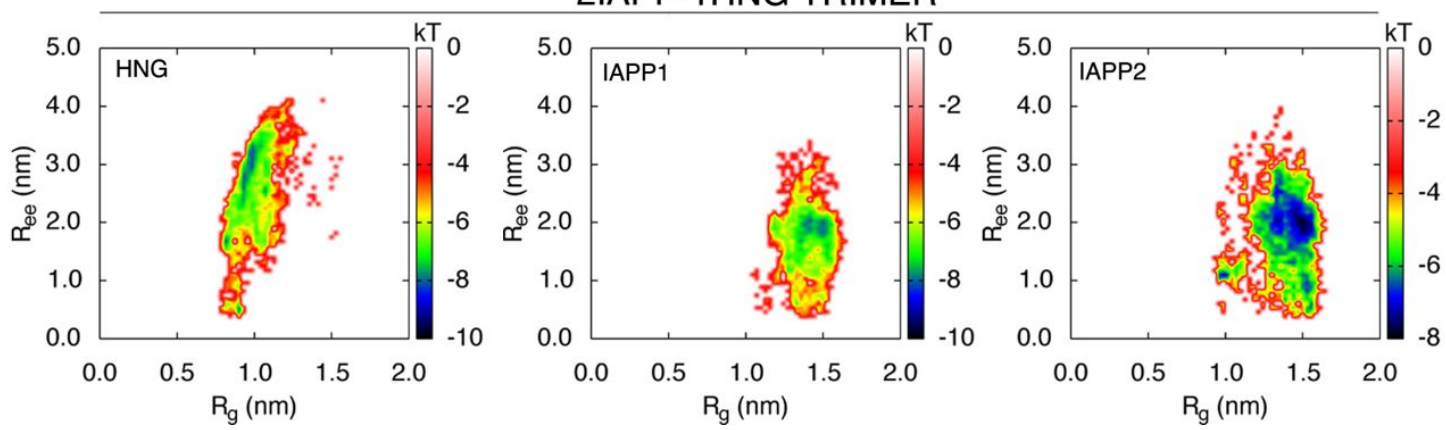

Figure S1. Free energy landscapes (FELs) from REMD reveal low-lying conformational basins that stabilize energy-minimized protein states (in blue) as a function of the protein end-to-end distance $\left(R_{e e}\right)$ and radius of gyration $\left(R_{g}\right)$. Mixing of HNG and IAPP proteins do not substantially modify the conformational ensembles that each protein inhabits. 

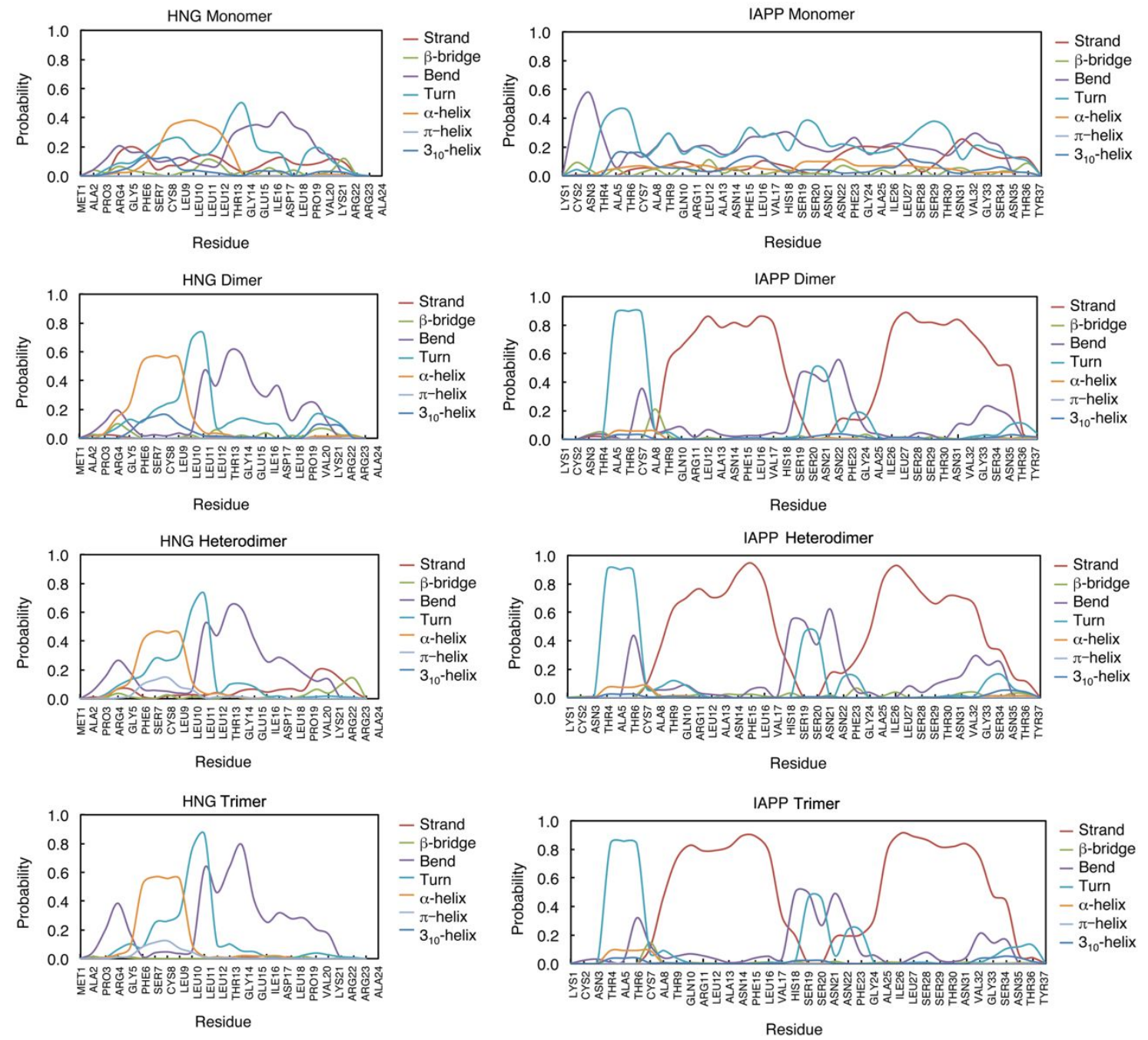

Figure S2. Dominant secondary structures for HNG (left) and IAPP (right) in monomeric (top row), homodimeric (second row), heterodimeric (third row), and trimeric (bottom row) states. IAPP $\beta$-strands are immediately stabilized in the presence of neighbors, however HNG secondary structures are only moderately stabilized when the protein is bound to neighbors. 
HNG MONOMER

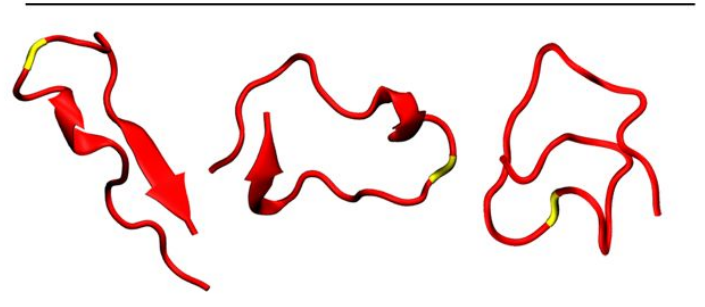

HNG DIMER

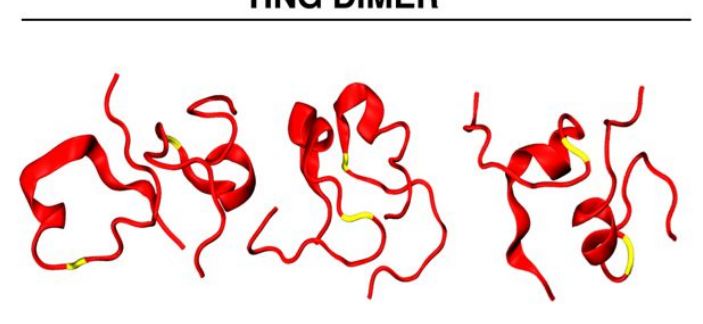

HNG-IAPP DIMER

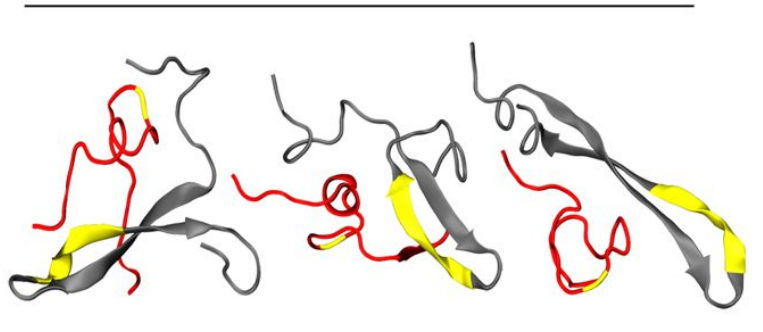

IAPP MONOMER

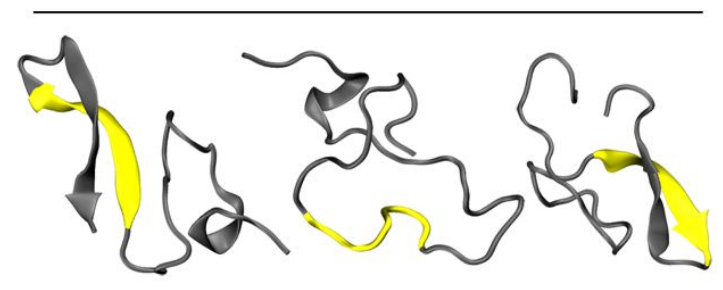

IAPP DIMER

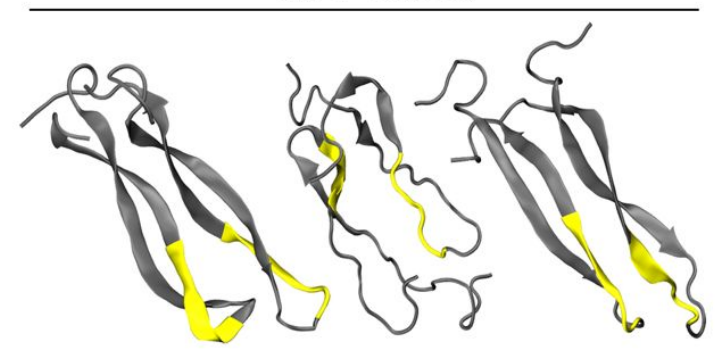

IAPP-HNG-IAPP TRIMER

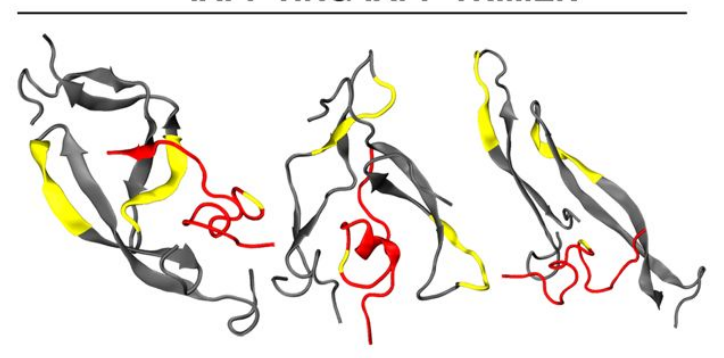

Figure S3. The HNG Gly14 residue and the IAPP-NFGAIL group (highlighted in yellow) are shown in the most dominant protein conformations. Gly14 (mutated from Ser14) does not appear to significantly affect the resulting HNG (red) morphologies. However, the IAPP (gray) NFGAIL region, which has been associated with pathological IAPP aggregation, is usually paired during IAPP dimerization, but is separated upon insertion of HNG. 
HNG MONOMER

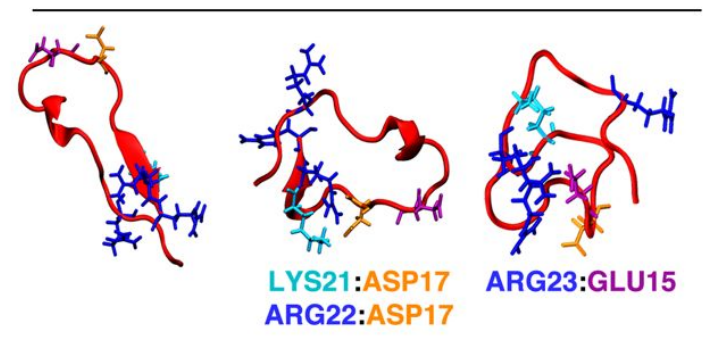

HNG DIMER

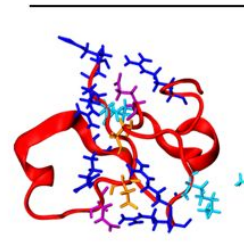

H1-GLU15:H2-ARG23

H1-ASP17:H2-ARG23

H2-ASP17:H2-ARG22

H2-GLU15:H1-ARG22

H2-GLU15:H2-ARG4

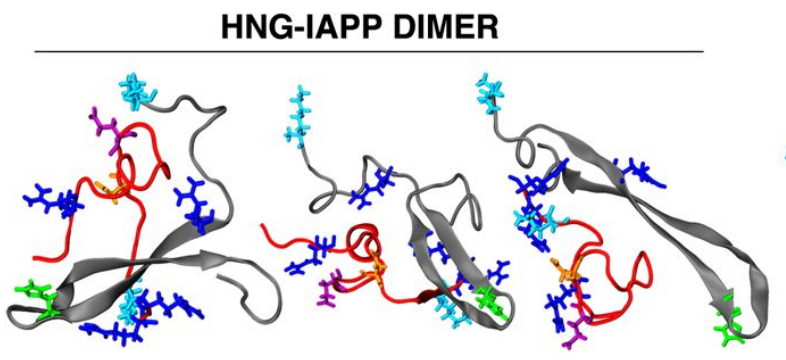

H-GLU15:I-LYS1 H-ARG4:H-GLU15 H-ASP17:H-ARG22 H-ARG4:H-GLU15

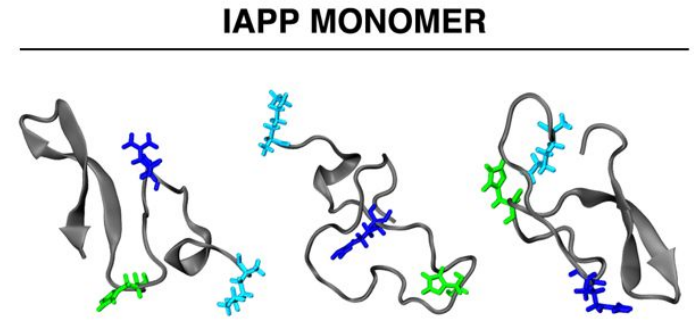

IAPP DIMER

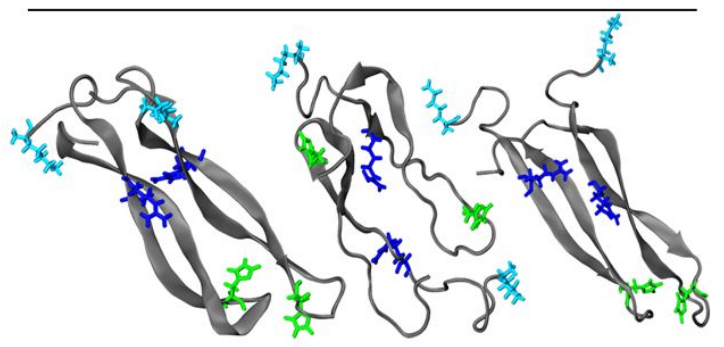

IAPP-HNG-IAPP TRIMER

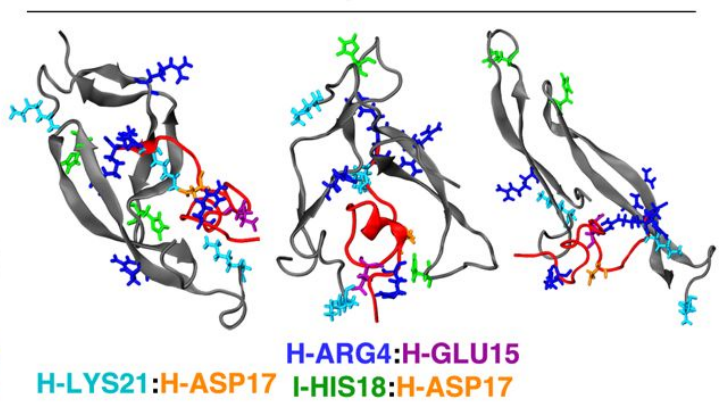

Figure S4. Salt bridges and electrostatic interactions between charged amino acids are highlighted for each of the dominant HNG (red) and IAPP (gray) protein clusters. While a large number of intra- and inter-molecular salt bridges exist in HNG dimers, heterodimers and trimers are not significantly stabilized by electrostatic interactions, but rather through hydrogen-bonding. 
HNG

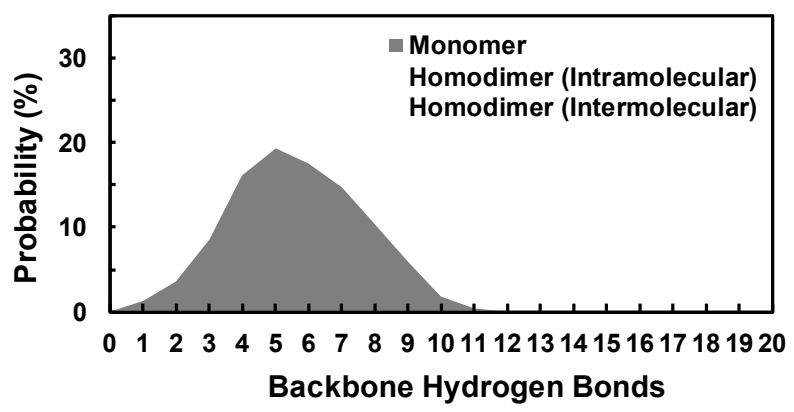

HNG+IAPP

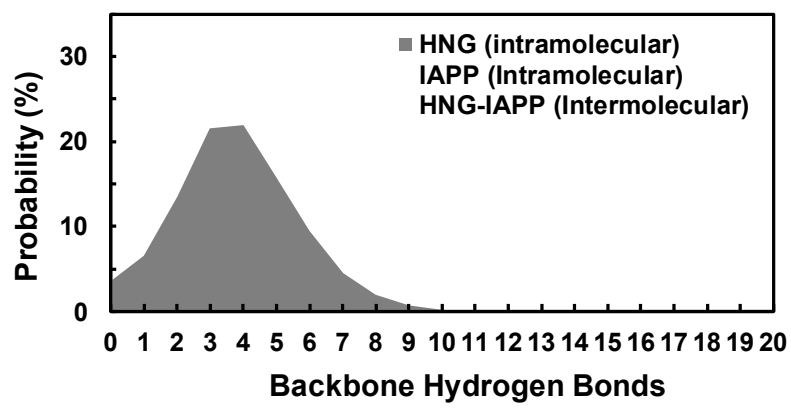

IAPP

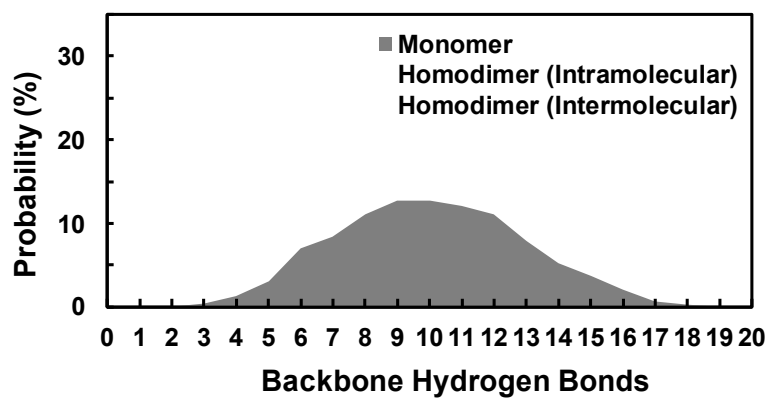

HNG+IAPP+IAPP

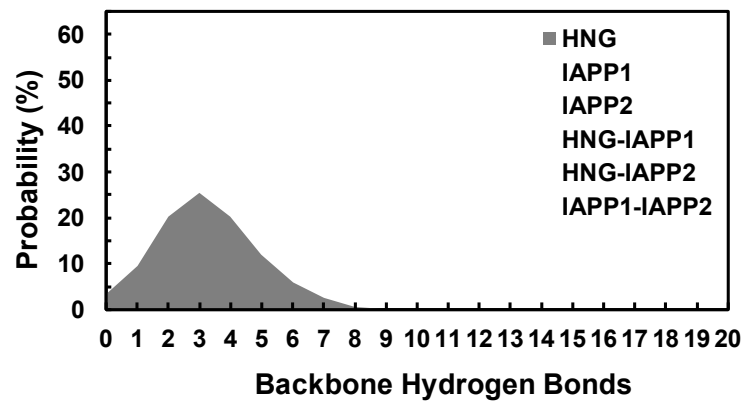

Figure S5. REMD simulations reveal the distributions of intra- and inter-molecular hydrogen bonds formed between protein backbones. IAPP monomers tend to exhibit more backbone hydrogen bonds per residue $(0.25)$ compared to HNG monomers $(0.20)$. Upon dimerization, HNG homodimers convert one backbone intramolecular hydrogen bond to an intermolecular backbone hydrogen bond while IAPP homodimers gain two intermolecular and four intramolecular hydrogen bonds. These trends are also observed in heterogeneous oligomers. 

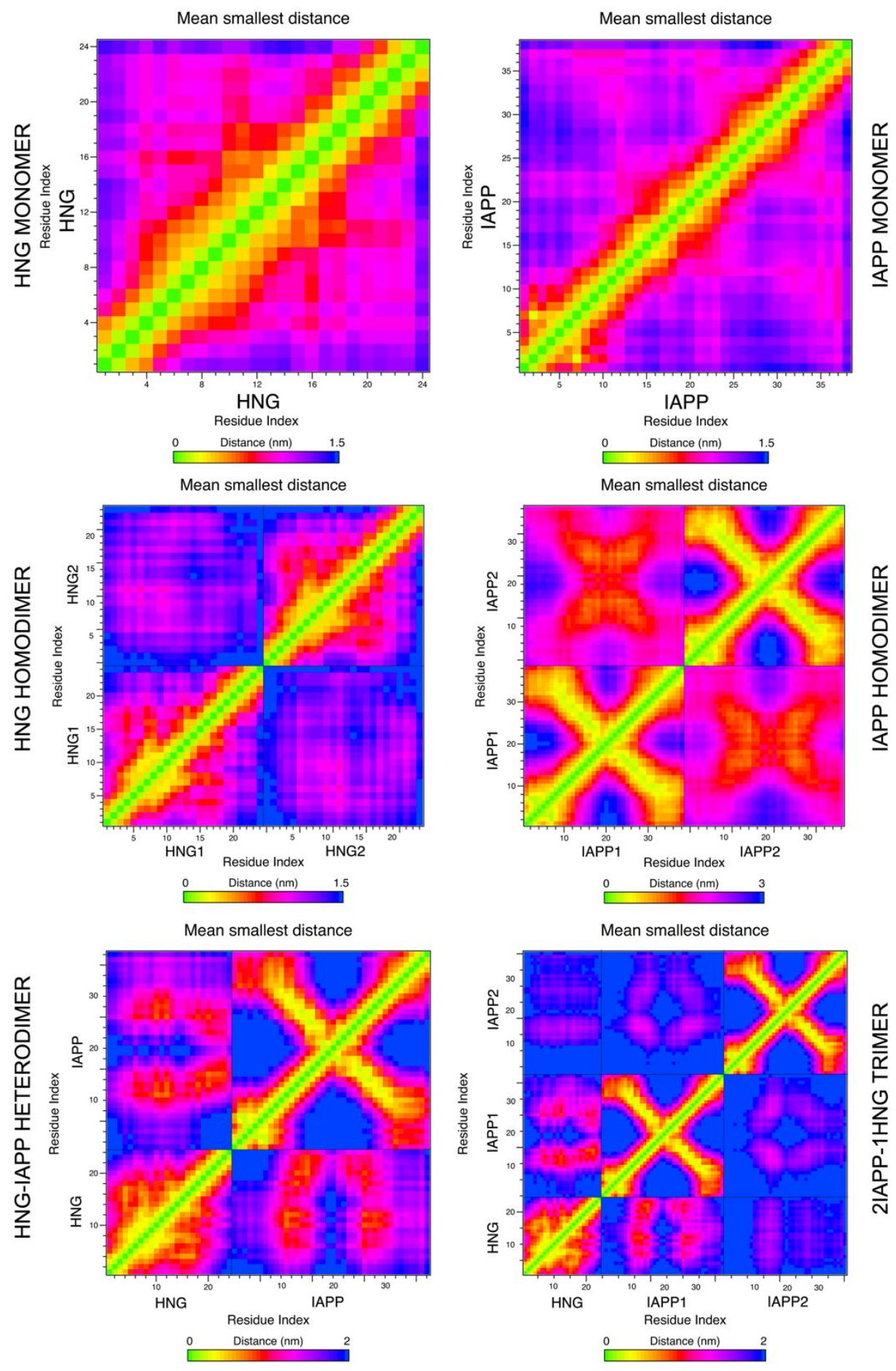

Figure S6. Protein contact maps from REMD simulations. Monomers exhibit very little intramolecular association, however intermolecular contact maps show close contact between ${ }_{12} \mathrm{LANFLV}_{17}$ and ${ }_{22} \mathrm{NFGAIL}_{27}$ regions in IAPP, representing a combination of parallel and antiparallel strands. Heterodimer contact maps reveal an association between $\mathrm{HNG}_{9} \mathrm{LLLL}_{12}$ and IAPP- $_{-11} \mathrm{RLA}_{13}$ or IAPP- ${ }_{26} \mathrm{ILS}_{28}$, and $\mathrm{HNG}_{-20} \mathrm{VKR}_{22}$ and IAPP- ${ }_{13} \mathrm{ANF}_{15}$ or IAPP- ${ }_{25} \mathrm{AIL}_{27}$. 


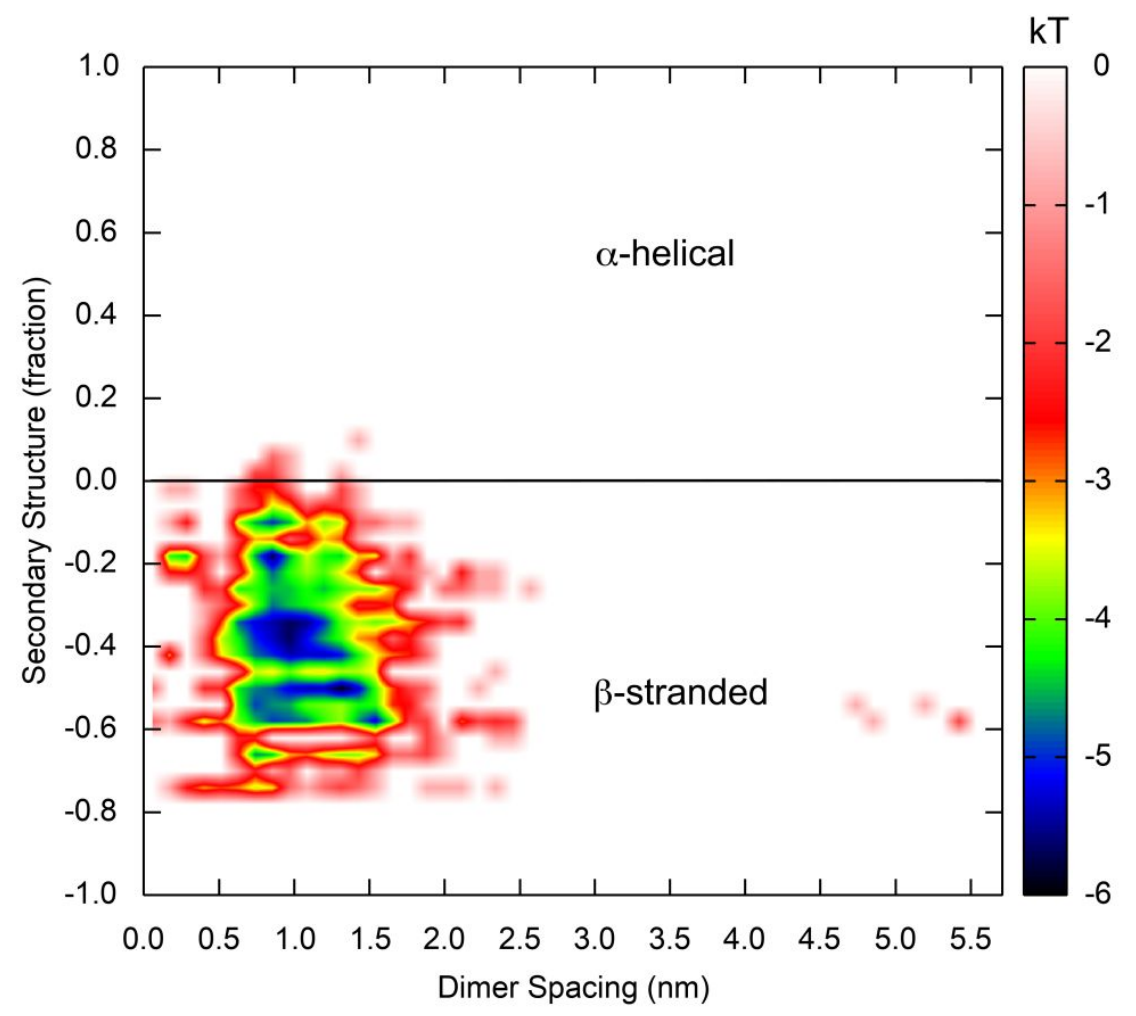

Figure S7. A free energy landscape showing IAPP populations in HNG-bound heterodimers for the entire REMD simulation. Secondary structure fraction (y-axis) refers to the fraction of IAPP residues that are alpha-helical (positive) vs. beta-stranded (negative). As a whole, IAPP tends to form HNG heterodimers with $20-60 \%$ of its residues exhibiting beta-strands. While bound IAPP can have fewer beta-stranded residues, nearly every sampled heterodimer conformation contains at least some IAPP beta-strands. 